\title{
An exploration of testosterone levels in patients with bipolar disorder
}

\author{
Sarah C. Wooderson, Peter Gallagher, Stuart Watson and Allan H. Young
}

\section{Background}

Testosterone influences well-being, mood and cognition and may play a role in the pathophysiology of bipolar disorder.

\section{Aim}

To examine testosterone levels in patients with bipolar disorder compared with healthy controls.

\section{Method}

We examined baseline total testosterone levels and current depression scores in male and female patients with bipolar disorder and mild to moderate depression and healthy controls.

\section{Results}

A significant interaction between diagnosis and gender was observed $(F(2,97)=9.791, P=0.002)$. Testosterone levels were significantly lower for male patients with bipolar disorder compared with male controls $(P=0.001)$. Women with bipolar disorder had significantly higher testosterone levels than female controls $(P=0.03)$

\section{Conclusions}

Disturbances in testosterone levels may represent an important neurobiological abnormality in bipolar disorder and may differ by gender. If these findings are confirmed, the use of gender appropriate treatment strategies for the normalisation of testosterone levels in bipolar disorder depression should be further explored.

\section{Declaration of interest}

None.

\section{Copyright and usage}

(c) The Royal College of Psychiatrists 2015. This is an open access article distributed under the terms of the Creative Commons Non-Commercial, No Derivatives (CC BY-NC-ND) licence.
Bipolar disorder is burdensome for the individual and for society: it is associated with significant functional impairment, high comorbidity, lower quality of life and higher rates of suicide. ${ }^{1}$ Pharmacotherapy with mood stabilisers and antipsychotics may not lead to sustained remission and more than two-thirds of those treated experience a recurrence within 5 years. ${ }^{2}$ Given such poor treatment outcomes, novel approaches to improve therapeutic interventions are an important objective of bipolar disorder research. Mounting, albeit inconsistent, evidence suggests that testosterone may play a role in the pathophysiology of mood disorders. ${ }^{3}$ However, the effects of testosterone are complex: the hormonal disorder polycystic ovarian syndrome is characterised by chronically increased testosterone levels and is associated with significantly increased depression, ${ }^{4}$ but paradoxically, amelioration of depression has been found following testosterone administration in males ${ }^{5}$ and females; ${ }^{6-8}$ low testosterone levels have been reported in certain subgroups of men with depression; ${ }^{9}$ androgen-induced hypomania has been reported; ${ }^{10}$ and high testosterone levels may predict suicidal behaviour in women with bipolar disorder. ${ }^{3}$ Further evidence clarifying the relationship between testosterone and bipolar disorder might pave the way for exploring the use of pharmacological strategies that optimise testosterone levels in patients with bipolar disorder. We therefore assessed testosterone levels in bipolar disorder and healthy controls and sought to explore whether this was different between males and females and whether there was a relationship between testosterone, mood and cognition.

\section{Method}

\section{Participants and study setting}

Participants in this study were 49 patients (male: $n=30$; female: $n=19)$ with a diagnosis of bipolar disorder ( $n=27$ bipolar disorder I; $n=22$ bipolar disorder II) and a current depressive episode (confirmed with the Structured Clinical Interview for DSM-IV ${ }^{11}$ ), who were 37 years old, and 37 education-matched healthy controls (male: $n=23$; female: $n=14$ ). Here we report baseline data collected as part of a study examining the effects of a glucocorticoid receptor antagonist in bipolar disorder depression. ${ }^{12}$ Patient medication was unchanged for 4 weeks before participation. After a complete description of the study, written informed consent was obtained from all participants. The study was carried out at Newcastle University in the north east of England and was approved by Newcastle and North Tyneside Local Research Ethics Committee.

\section{Neuroendocrine assessment}

Blood samples were taken at $15.00 \mathrm{~h}$. The blood was spun at $3000 \mathrm{rpm}$ for $10 \mathrm{~min}$ in a refrigerated centrifuge, then separated and stored at $-80^{\circ} \mathrm{C}$ until assayed for total testosterone. Testosterone was measured using the Roche Testosterone II assay on the Roche Modular Analytics E 170 analyser. Internal quality control involved material being assayed twice a day. The Clinical Biochemistry team at Newcastle Hospitals participates in the UK National External Quality Assessment Scheme (NEQAS) Steroid External Quality Assessment (EQA) Scheme.

\section{Mood symptoms}

The following mood measures were administered: the Hamilton Rating Scale for Depression (HRSD), ${ }^{13}$ the Montgomery and Åsberg Depression Rating Scale (MADRS) ${ }^{14}$ and the Beck Depression Inventory (BDI). ${ }^{15}$

\section{Statistical analyses}

Data were analysed using SPSS software (version 22 for Windows). Baseline demographic, neuroendocrine and mood data were compared between patients with bipolar disorder and controls. Due to the normal distribution of the data, quantitative characteristics are reported using means and standard deviations. We report summaries for the characteristics and the results of comparisons using independent-samples $t$-tests. Differences between gender (male $v$. female) and diagnosis (control $v$. patient) 
Table 1 Demographic and clinical features of patients with bipolar disorder compared with healthy controls

\begin{tabular}{|c|c|c|c|c|}
\hline & Male patients $(n=30)$ & Male controls $(n=23)$ & Female patients $(n=19)$ & Female controls $(n=14)$ \\
\hline Demographic and clinical features ${ }^{a}$ & \multicolumn{4}{|c|}{ Mean (s.d.) } \\
\hline Age, years & $47(10.0)$ & 42 (15.4) & $47(9.1)$ & $48(10.7)$ \\
\hline HDRS & $18(4.6)$ & $0.1(0.3)$ & $21(4.6)$ & $0.1(0.4)$ \\
\hline MADRS & $26(8.7)$ & $0.5(0.9)$ & $28(8.5)$ & $0.4(0.7)$ \\
\hline $\mathrm{BDI}$ & $27(10.5)$ & $0.9(1.5)$ & $30(11.3)$ & $0.9(1.2)$ \\
\hline Testosterone level, nmol/L & $11.3(3.8)$ & $15.5(4.2)$ & $1.4(0.4)$ & $1.2(0.2)$ \\
\hline
\end{tabular}

in terms of testosterone levels were examined using as two-way analysis of variance (ANOVA). All cited $P$-values are two-tailed, with a significance level set at $P=0.05$.

\section{Results}

Demographic and clinical features of male and female patients with bipolar disorder compared with healthy male and female controls are outlined in Table 1.

A two-way ANOVA revealed a statistically significant interaction between gender and diagnosis on baseline testosterone level, $F(2,97)=9.791, P=0.002$. These results together with the independent-samples $t$-test indicated that male participants with bipolar disorder had reduced testosterone levels compared with male controls, $t(51)=3.777, P=0.001$, whereas female participants with bipolar disorder had higher testosterone levels compared with female controls, $t(31)=-2.252, P=0.03$.

No significant correlations were observed between total testosterone levels and depression severity on the HRSD, MADRS or the BDI in patients. We found no relationship between testosterone and neuropsychological performance.

\section{Discussion}

To our knowledge, this is one of the first studies to directly investigate differences in testosterone levels in participants with current depression with bipolar disorder compared with healthy matched controls. Testosterone levels were found to be significantly lower in male patients with bipolar disorder depression compared with male controls, whereas female participants with bipolar disorder depression had significantly higher testosterone levels than female controls.

These findings support earlier work showing low testosterone in males with depression. ${ }^{8}$ In a recent study examining whether testosterone predicted suicide attempts in females with bipolar disorder followed up prospectively for up to 2.5 years, ${ }^{3}$ the authors reported that higher baseline testosterone levels predicted suicide attempts during the follow-up period. Although the present study was not a follow-up study and we did not investigate links with suicide, we do report a consistent finding of abnormal testosterone levels being significant also for females with bipolar disorder with current depression.

To explore this finding further with a view to establishing new treatments for bipolar disorder, further research is necessary to confirm the role of testosterone in bipolar disorder and to understand why this divergence in testosterone levels occurs in males and in females with the illness. Important clinical questions to explore in this field of research from both a biological and psychological perspective are: (a) whether reduced anabolic or androgenic effects caused by low levels of testosterone reduce feelings of masculinity and therefore self-satisfaction and self-worth in males; (b) whether an increase in the anabolic or androgenic effects of testosterone reduce feelings of femininity, lowering selfsatisfaction and self-worth in females; (c) whether testosterone could be a useful biomarker for bipolar disorder; (d) whether testosterone could be a useful biomarker for depression per se; and (e) whether testosterone levels are stable in the course of bipolar disorder depression or could they reflect a worsening of bipolar disorder symptoms, including signifying risk of suicide in females with bipolar disorder. ${ }^{3}$ Additionally, a systematic review of the effects of testosterone treatments would be important before testosterone normalisation treatments are recommended.

\section{Strengths and limitations}

The study was relatively small and exploratory. We enrolled only participants with bipolar disorder with current depression into the study, therefore our findings may only relate to participants in this particular phase of the illness. The strength of the study was that the sample was clinically well characterised.

\section{Implications}

Pharmacological interventions for bipolar disorder depression can be effective, yet many patients fail to respond to treatment. The findings presented herein build on an existing literature that draws attention to the role of testosterone in bipolar disorder and has significant implications for clinical interventions. Expanding treatment methods by incorporating hormonal monitoring may reflect an important shift in addressing the treatment of bipolar disorder using a more holistic approach. These preliminary findings should be examined further to establish the consequences of altered sex hormone levels in bipolar disorder depression.

\footnotetext{
Sarah C. Wooderson, MBPSS, BSC, MSC, PhD, Centre for Affective Disorders, Institute of Psychiatry, Psychology \& Neuroscience (IOPPN), King's College London, UK; Peter Gallagher, PhD, Institute of Neuroscience (Academic Psychiatry), Newcastle University, Newcastle, UK; Stuart Watson, MBBS, MD, MRCPsych, Institute of Neuroscience, Newcastle University, Newcastle, UK; Allan H. Young, MBChB, MPhil, PhD, FRCPsych, FRCPC, Centre for Affective Disorders, IOPPN, King’s College London, UK

Correspondence: Allan H. Young, Centre for Affective Disorders, Institute of Psychiatry, Psychology \& Neuroscience, King's College London, P072 De Crespigny Park, London SE5 8AF, UK. Email: allan.young@kcl.ac.uk

First received 19 May 2015, final revision 1 Sep 2015, accepted 16 Oct 2015
}

\section{Funding}

This work was supported by grant funding from the Stanley Medical Research Institute (REF: 03T-429) and the Medical Research Council (GU0401207). P.G. and S.W. received Research Capability Funding support from the Northumberland, Tyne and Wear NHS Foundation Trust. A.H.Y. and S.C.W. are supported by the National Institute for Health Research (NIHR) Biomedical Research Centre for Mental Health at South London and Maudsley NHS Foundation Trust and Institute of Psychiatry, Psychology \& Neuroscience, King's College London. This study presents independent research part-funded by the NIHR Biomedical 
Research Centre at South London and Maudsley NHS Foundation Trust and King's College London. The views expressed are those of the authors and not necessarily those of the NHS, the NIHR or the Department of Health. No external funding was used for this study.

\section{Acknowledgements}

We thank the participants who contributed to the research and to those clinicians involved in the wider research programme, including recruitment and screening: Nicol Ferrier, Nira Ahuja, Sankalpa Basu, Jane Carlile, Louise Golightly, Thiyyancheri Harikumar, Patrick Keown, Samer Makhoul, Anuradha Menon, Gavin Mercer, Rajesh Nair, Bruce Owen and Nanda Palanichamy. We also thank the Medical Research Council and the Stanley Medical Research Institute for supporting this work, and the Clinical Biochemistry Team at Newcastle Hospitals for measuring the testosterone levels.

\section{References}

1 Novick DM, Swartz HA, Frank E. Suicide attempts in bipolar I and bipolar I disorder: a review and meta-analysis of the evidence. Bipolar Disord 2010; 12 $1-9$

2 Sylvia LG, Thase ME, Reilly-Harrington NA, Salcedo S, Brody B, Kinrys G, et al. Psychotherapy use in bipolar disorder: association with functioning and illness severity. Aust NZ J Psychiatry 2015; 49: 453-61.

3 Sher L, Grunebaum MF, Sullivan GM, Burke AK, Cooper TB, Mann JJ, et al. Association of testosterone levels and future suicide attempts in females with bipolar disorder. J Affect Disord 2014; 166: 98-102.

4 Weiner $\mathrm{CL}$, Primeau $\mathrm{M}$, Ehrmann DA. Androgens and mood dysfunction in women: comparison of women with polycystic ovarian syndrome to healthy controls. Psychosom Med 2004; 66: 356-62.
5 Amanatkar HR, Chibnall JT, Seo BW, Manepalli JN, Grossberg GT. Impact of exogenous testosterone on mood: a systematic review and meta-analysis of randomized placebo-controlled trials. Ann Clin Psychiatry 2014; 26: 19-32.

6 Burd ID, Bachmann GA. Androgen replacement in menopause. Curr Womens Health Rep 2001; 1: 202-5.

7 Crammer JL. Premenstrual depression, cortisol and oestradiol treatment. Psychol Med 1986; 16: 451-5.

8 Studd J. Hormone therapy for reproductive depression in women. Post Reprod Health 2014; 20: 132-7.

9 Johnson JM, Nachtigall LB, Stern TA. The effect of testosterone levels on mood in men: a review. Psychosomatics 2013; 54: 509-14.

10 Freinhar JP, Alvarez W. Androgen-induced hypomania. J Clin Psychiatry 1985; 46 354-5.

11 First MB, Spitzer RL, Gibbon M, Williams JBW. Structured Clinical Interview for DSM-IV Axis I Disorders, Research Version. Biometrics Research, 1997.

12 Watson S, Gallagher P, Porter RJ, Smith MS, Herron L, Bulmer S, et al. A randomized trial to examine the effect of mifepristone on neuropsychological performance and mood in patients with bipolar depression. Biol Psychiatry 2012; 72: 943-9.

13 Hamilton M. A rating scale for depression. J Neurol Neurosurg Psychiatry 1960; 23: $56-62$.

14 Montgomery SA, Åsberg M. A new depression scale designed to be sensitive to change. Br J Psychiatry 1979; 134: 382-9.

15 Beck AT, Ward CH, Mendelson M, Mock J, Erbaugh J. An inventory for measuring depression. Arch Gen Psychiatry 1961; 4: 561-71. 